\title{
Steady compressible vortex flows: the hollow-core vortex array
}

\author{
By K. ARDALAN ${ }^{1}$, D. I. MEIRON ${ }^{1}$ AND D. I. PULLIN \\ ${ }^{1}$ Applied Mathematics, California Institute of Technology, Pasadena, CA 91125, USA \\ ${ }^{2}$ Graduate Aeronautical Laboratories, California Institute of Technology, Pasadena, \\ CA 91125, USA
}

(Received 28 December 1994 and in revised form 26 June 1995)

We examine the effects of compressiblity on the structure of a single row of hollowcore, constant-pressure vortices. The problem is formulated and solved in the hodograph plane. The transformation from the physical plane to the hodograph plane results in a linear problem that is solved numerically. The numerical solution is checked via a Rayleigh-Janzen expansion. It is observed that for an appropriate choice of the parameters $M_{\infty}=q_{\infty} / c_{\infty}$, and the speed ratio, $a=q_{\infty} / q_{v}$, where $q_{v}$ is the speed on the vortex boundary, transonic shock-free flow exists. Also, for a given fixed speed ratio, $a$, the vortices shrink in size and get closer as the Mach number at infinity, $\boldsymbol{M}_{\infty}$, is increased. In the limit of an evacuated vortex core, we find that all such solutions exhibit cuspidal behaviour corresponding to the onset of limit lines.

\section{Introduction}

Many practical problems of interest arising in physics, mathematics and engineering involve the study of vortices and vortex motions. The persistent trailing vortices behind jumbo jets constitute a hazard to other aircraft which has led to a study of vortex formation by the roll-up of vortex sheets and the decay and interaction of vortex pairs. Attempts to understand two-dimensional turbulence have led to studies of the statistical mechanics of random arrays of point vortices. The observations of the turbulent mixing layer (Roshko 1976) show convincing evidence that the layer consists of a row of quasi-two-dimensional coherent structures whose amalgamation into larger similar structures produces the growth of the mixing layer. Several theoretical studies attempt to model the formation of these and other structures by studying the interaction of two-dimensional vortices. Yet, these studies are almost exclusively for incompressible flow (Saffman \& Sheffield 1977; Huang \& Chow 1982) and few theoretical studies exist on the interaction of vortices in a compressible medium.

Ringleb (1940), Shapiro (1953), Mack (1960), and Brown (1965) are some of the earlier researchers whose study of the single compressible vortex highlighted the effects of compressibility on the structure of the vortex core as well as its stability characteristics. Moore (1985) studied the effects of compressibility on the speed of a vortex ring. Subsequently, Moore \& Pullin 1987 succeeded in constructing the flow field of a translating vortex pair in compressible irrotational flow by transforming the problem to the hodograph plane. In their formulation, each vortex was modelled by a constant-pressure stagnant core surrounded by a closed vortex sheet. They realized 
that for free-boundary problems of this type, this transformation was effective because it fixed the vortex sheet boundary in the hodograph plane, hence circumventing the need for curvilinear grids.

Our aim is to study the basic interactions of vortices in a compressible fluid via a search for steady solutions of the Euler equations corresponding to flows with concentrated vortex cores. An infinite array of point vortices is the simplest model for a shear layer in incompressible flow. It is well known that a hollow-core vortex in a compressible medium is the limit of a point vortex in an incompressible medium. Hence, we choose to construct the compressible analogue of the single row vortex array and to study the effects of compressibility on this model. In this investigation it is important to identify the parameter space which will admit shock-free transonic flow.

\section{The physical plane}

We consider an infinite linear array of identical vortices lying on the $x$-axis each separated by a distance $L$ measured from the vortex centre. Each vortex is hollow (i.e. stagnant, constant-pressure core.) Since the flow is steady and the pressure is constant inside the cores, the fluid speed on the boundary of the vortex must be a constant value, $q_{v}$. Outside the cores, the flow is that of an ideal gas and it is assumed to be irrotational, compressible and homentropic. The vortices have the same circulation, $\Gamma$, such that at large distances the flow becomes asymptotic to that produced by a vortex sheet of strength $2 U_{\infty}$ lying along the $x$-axis.

We shall seek steady solutions of the configuration sketched in figure 1 in which the vortices have fore-and-aft symmetry, i.e. each vortex is symmetrical about the $x$-axis and a line parallel to the $y$-axis through the vortex centre. Hence, it will be sufficient to investigate the details of the flow in the geometry depicted in figure 2 . The equation of continuity in the case of steady two-dimensional compressible flow can be written as

$$
\frac{\partial(\rho u)}{\partial x}+\frac{\partial(\rho v)}{\partial y}=0 .
$$

We can satisfy this identically by introducing a stream function $\psi$ defined so that

$$
\rho u=-\rho_{o} \frac{\partial \psi}{\partial y}, \quad \rho v=\rho_{o} \frac{\partial \psi}{\partial x},
$$

where $\rho_{o}$ is any reference density. We shall identify the density $\rho_{o}$ with the density of the flow at infinity $\left(\rho_{\infty}\right)$ and set $\rho_{\infty}=1$ without loss of generality.

The governing equation in this problem is given by Bernoulli's theorem. For homentropic flow of an ideal gas, if $c$ is the speed of sound and $p$ is the pressure, we have $c^{2}=\gamma p / \rho$ and therefore conservation of energy requires

$$
\frac{c^{2}}{\gamma-1}+\frac{1}{2} q^{2}=\frac{c_{s}^{2}}{\gamma-1},
$$

where $q$ is the speed of the flow and $c_{s}$ is the speed of sound at the stagnation condition (i.e. when $q=0$.)

We seek the solution of these equations in the computational domain depicted in figure 2 , which shows a sketch of the streamlines. The point $S$ corresponds to the stagnation point where the speed of the fluid $q$ is zero. The curve connecting points $A$ and $B$ represents the boundary of the vortex. The vertical lines from $B$ and $S$ are 


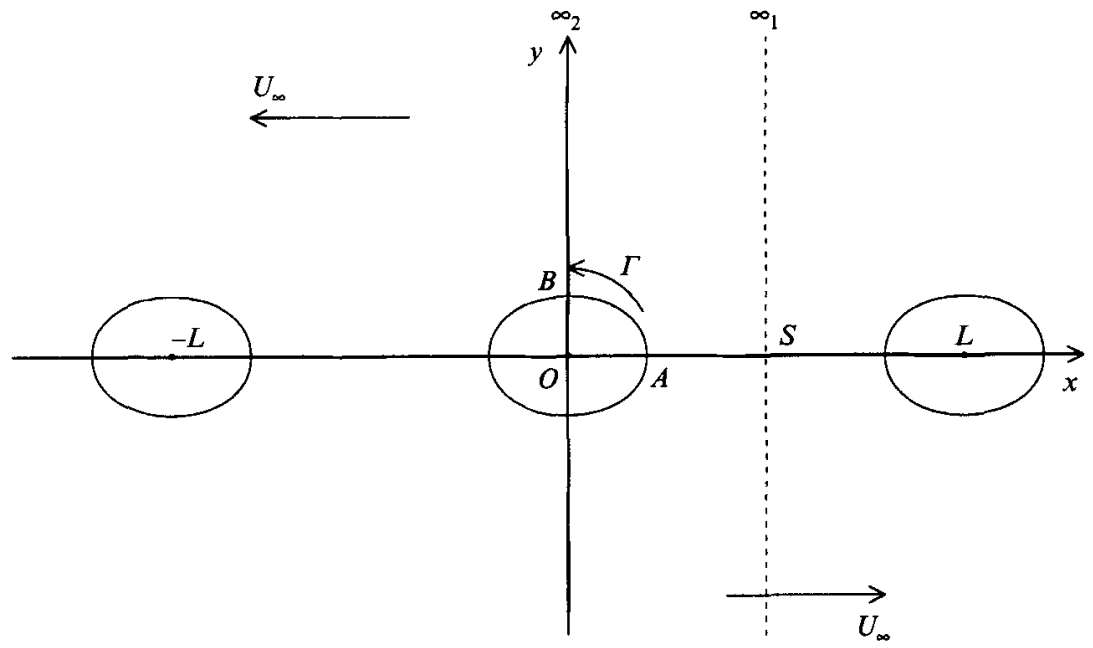

FIGURE 1. A sketch of the problem in the physical space. The closed curves show the vortex boundaries on which the speed is $q_{v}$.

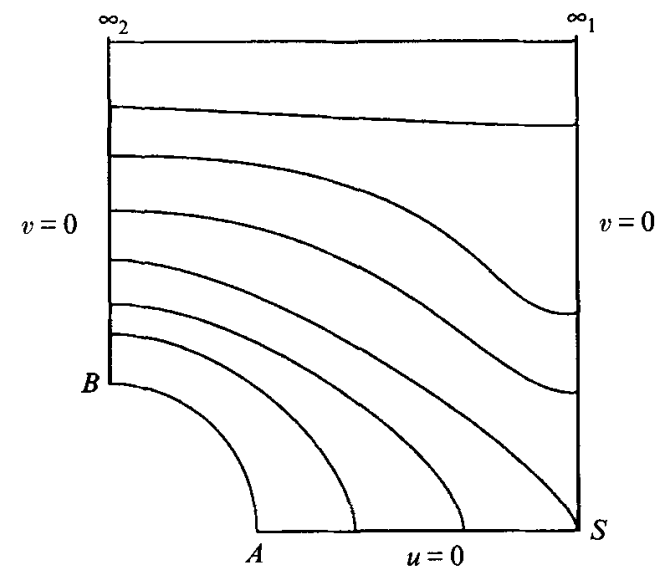

FIGURE 2. A description of the computational domain in physical space.

assumed to extend to infinity where the velocity of the flow has a single speed, $q$, and phase direction, $\theta$, namely, $q=q_{\infty}$ and $\theta=\pi$. On the two vertical boundaries $\infty_{1}-S$ and $B-\infty_{2}$, even though the speed of the flow varies, the assumed symmetries determine the phase angle of the velocity vector to be $\theta=\pi$, whilst on the symmetry line $S-A$, the flow angle is $\theta=\pi / 2$. Even though it is possible to proceed with the solution of this problem in the physical plane, we instead transform to the hodograph plane where the geometry of the flow simplifies to a rectangle and the governing equation is Chaplygin's equation, which is a linear equation for the stream function.

\section{The hodograph plane}

\subsection{Chaplygin's equation}

The velocity potential of an irrotational compressible flow satisfies a non linear partial differential equation. When $(q, \theta)$ are taken as variables, the equation becomes linear (Von Mises 1958). This leads to a quasi-conformal map from the physical plane, $(x, y)$-space, to the hodograph plane, $(q, \theta)$-space, which we shall summarize for 
completeness. Along with the stream function $\psi$ defined earlier we can introduce the velocity potential $\phi$ and write

$$
\left[\begin{array}{c}
-\mathrm{d} \phi \\
\mathrm{d} \psi\left(-\rho_{o} / \rho\right)
\end{array}\right]=\left[\begin{array}{cc}
u & v \\
-v & u
\end{array}\right]\left[\begin{array}{l}
\mathrm{d} x \\
\mathrm{~d} y
\end{array}\right]
$$

and using methods of complex variables it is easily verified (Milne-Thomson 1966) that

$$
\mathrm{d} z=-\frac{\mathrm{e}^{\mathrm{i} \theta}}{q}\left(\mathrm{~d} \phi+\mathrm{i} \frac{\rho_{o}}{\rho} \mathrm{d} \psi\right),
$$

where $z=x+\mathrm{i} y$ and $u-\mathrm{i} v=q \mathrm{e}^{\mathrm{i} \theta}$. It follows that

$$
\begin{aligned}
& \frac{\partial z}{\partial q}=-\frac{\mathrm{e}^{\mathrm{i} \theta}}{q}\left(\frac{\partial \phi}{\partial q}+\mathrm{i} \frac{\rho_{o}}{\rho} \frac{\partial \psi}{\partial q}\right), \\
& \frac{\partial z}{\partial \theta}=-\frac{\mathrm{e}^{\mathrm{i} \theta}}{q}\left(\frac{\partial \phi}{\partial \theta}+\mathrm{i} \frac{\rho_{o}}{\rho} \frac{\partial \psi}{\partial \theta}\right) .
\end{aligned}
$$

Cross-differentiation of the above equations then leads to a compatibility condition akin to the Cauchy-Riemann equations:

$$
\frac{\partial \phi}{\partial q}=q \frac{\partial \psi}{\partial \theta} \frac{\partial}{\partial q}\left(\frac{1}{\rho q}\right)
$$

and

$$
\frac{\partial \phi}{\partial \theta}=\frac{q}{\rho} \frac{\partial \psi}{\partial q}
$$

These are the equations of the hodograph plane. Eliminating $\phi$ from equations (3.4) and (3.5) and noting that, in the hodograph plane, the density is a function of the speed only, $\rho=\rho(q)$, leads to Chaplygin's equation,

$$
q^{2}\left[1-\frac{q^{2}(\gamma-1)}{2 c_{s}^{2}}\right] \frac{\partial^{2} \psi}{\partial q^{2}}+q\left[1-\frac{q^{2}(\gamma-3)}{2 c_{s}^{2}}\right] \frac{\partial \psi}{\partial q}+\left[1-\frac{q^{2}(\gamma+1)}{2 c_{s}^{2}}\right] \frac{\partial^{2} \psi}{\partial \theta^{2}}=0 .
$$

\subsection{Scaling of variables}

We next consider the image of our problem in the hodograph plane. Before doing so we scale all speeds by the speed of the vortex boundary, $q_{v}$. Furthermore, we shall scale the stream function by the strength of the singularity at infinity in the hodograph plane, which we denote by $\mathscr{A}$. This along with our earlier choice of the reference density, namely $\rho_{\infty}=1$, leads to the following relationship between the length, time and mass scales $L^{\prime}, T^{\prime}, M^{\prime}$ :

$$
L^{\prime 3} \rho_{\infty} M^{\prime-1}=1, \quad q_{v} T^{\prime} L^{\prime-1}=1, \quad \mathscr{A} L^{\prime-2} T^{\prime}=1 .
$$

At this point we have completed the specification of scales for our problem. It is important to note here that the distance between the vortices $L$ has not been used for scaling and that it shall be determined as part of the solution.

The use of equation (2.2) allows us to express the speed of sound at the stagnation point as

$$
\frac{1}{c_{s}^{2}}=\frac{2 M_{\infty}^{2}}{2+(\gamma-1) M_{\infty}^{2}} \frac{1}{a^{2} q_{v}^{2}}
$$

where $a=q_{\infty} / q_{v}$ and $M_{\infty}=q_{\infty} / c_{\infty}$, the Mach number at infinity. We shall define 


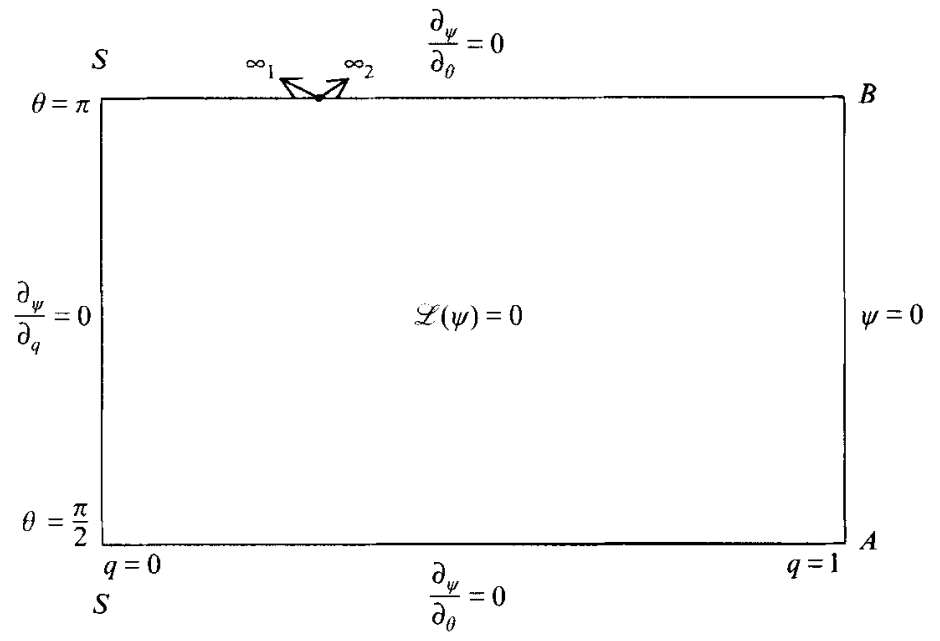

FIGURE 3. Computational domain in the hodograph plane.

$\beta=q_{v}^{2} / 2 c_{s}^{2}$ and hence can re-write Chaplygin's equation as follows:

$$
q^{2}\left[1-\beta(\gamma-1) q^{2}\right] \psi_{q q}+q\left[1-\beta(\gamma-3) q^{2}\right] \psi_{q}+\left[1-\beta(\gamma+1) q^{2}\right] \psi_{\theta \theta}=0 .
$$

\subsection{The flow in the hodograph plane}

To determine the image of the flow in the hodograph plane we must return to figure 2. Since at the stagnation point $S$ the speed is zero, the streamlines form a saddle point pattern at which the flow angle takes all values between $\pi / 2$ and $\pi$. Therefore, the stagnation point maps into the vertical line $q=0$ in figure 3 . Moving on to the boundary of the vortex, we know that the speed is constant $\left(q=q_{v}\right)$, so after scaling by $q_{v}$, the vertical line $q=1$ is the image of the vortex boundary in the hodograph plane. The streamline at infinity corresponds to a single speed, $q=q_{\infty}$, and direction, $\theta=\pi$, and so we see that an entire streamline is transformed into a point singularity in the hodograph plane. We defer a discussion of the nature of this singular tranformation for the moment and discuss boundary conditions.

Since the stream function is arbitrary to within a constant, we will choose $\psi=0$ on the boundary of the vortex without loss of generality. On the boundary $A-S$, we have $u=0$. Additionally, figure 2 shows that $\mathrm{d} y=0$. Using this information along with equations (3.1), (3.4) and (3.5) we obtain that $\partial \psi / \partial \theta=0$ on $\theta=\pi / 2$. On the segment from $B-\infty_{2}$ and $\infty_{1}-S$, we have $v=0$ and the streamlines have a single direction $\theta=\pi$. It is also clear that here $\mathrm{d} x=0$. The use of the hodograph transformation then leads us to the condition that $\partial \psi / \partial \theta=0$ on $\theta=\pi$. Having determined these boundary conditions, we can now investigate the nature of the solution near the stagnation point. An asymptotic analysis of Chaplygin's equation near $q=0$ shows that $\psi \sim c_{1}+c_{2} q^{2} \cos (2 \theta)+O\left(q^{4}\right)$ where $c_{1}$ and $c_{2}$ are constants. Hence, setting $\partial \psi / \partial q=0$ on $q=0$ is consistent. The problem to be solved in the hodograph plane is depicted in figure 3 .

\subsection{The singularity at infinity}

We assume that the flow at infinity is subsonic since otherwise a shock-free flow field seems unlikely (Moore \& Pullin 1987.) Assuming small disturbances at infinity and distorting the incompressible flow solution for a row of equal point vortices as 
suggested by Prandtl-Glauert theory we find that the velocity potential is

$$
\phi=\frac{\Gamma}{2 \pi} \arctan \left[\cot (\pi x / L) \tanh \left(\pi\left(1-M_{\infty}^{2}\right)^{1 / 2} y / L\right)\right] .
$$

To leading order, therefore, the stream function is given by

$$
\psi=\frac{\Gamma}{2 L} y
$$

and the corresponding velocity field is

$$
u=\frac{\Gamma}{2 L} \frac{\sinh \left(2 \pi\left(1-M_{\infty}^{2}\right)^{1 / 2} y / L\right)}{\cosh \left(2 \pi\left(1-M_{\infty}^{2}\right)^{1 / 2} y / L\right)-\cos (2 \pi x / L)}
$$

and

$$
v=\frac{\Gamma\left(1-M_{\infty}^{2}\right)^{1 / 2}}{2 L} \frac{\sin (2 \pi x / L)}{\cosh \left(2 \pi\left(1-M_{\infty}^{2}\right)^{1 / 2} y / L\right)-\cos (2 \pi x / L)} .
$$

In order to find the nature of the singularity in the hodograph plane we need to eliminate $x$ and $y$ from (3.10), (3.11) and (3.12). Using local quasi-polar coordinates $(s, \delta)$ centred at the point at infinity in the hodograph plane defined by

$$
\mathrm{se}^{\mathrm{i} \delta}=(q-a)+\mathrm{i} \frac{\theta-\pi}{\left(1-M_{\infty}^{2}\right)^{1 / 2}},
$$

we find that, for small $s$, the leading-order stream function is given by

$$
\psi=\mathscr{A} \log (s)
$$

This form of the singularity indicates that as $s \rightarrow 0$, regardless of direction $\delta$, all higher-order corrections to the logarithm go to zero. We have now posed the problem in the hodograph plane. Its solution $\psi(q, \theta)$ is a two-parameter family depending on $a=q_{\infty} / q_{v}$, the location of the singularity in the hodograph plane and $M_{\infty}=q_{\infty} / c_{\infty}$, the Mach number at infinity.

\section{The incompressible problem}

We begin by obtaining an analytical solution in the hodograph plane for the $M_{\infty}=0$ limit of the single row. Setting $M_{\infty}=0$ reduces (3.8) to Laplace's equation so by using equation (3.14) we deduce that the correct form of the singular solution near the point at infinity is given by

$$
\psi_{s}=\log \left(q^{2}+a^{2}+2 a q \cos \theta\right)
$$

Now all that is necessary to complete the solution is to satisfy the boundary conditions. We apply the method of images to obtain the solution

$$
\psi_{o}=\log \left[\frac{\left(q^{2}+a^{2}+2 a q \cos \theta\right)\left(q^{2}+a^{2}-2 a q \cos \theta\right)}{\left(q^{2} a^{2}+1+2 a q \cos \theta\right)\left(q^{2} a^{2}+1-2 a q \cos \theta\right)}\right] .
$$

This solution agrees with that given by Baker, Saffman \& Sheffield (1976) who solved the same problem using the ideas of free-streamline theory. For comparison, we find a parametric representation for the vortex boundary centred at the origin. The non-dimensional coordinates of the boundary are given by

$$
x=-2 \frac{a^{2}+1}{a}\left[\tan ^{-1}\left[\frac{(a+1) \sin \theta}{(\cos \theta+1)(a-1)}\right]-\tan ^{-1}\left[\frac{(a-1) \sin \theta}{(\cos \theta+1)(a+1)}\right]\right],
$$




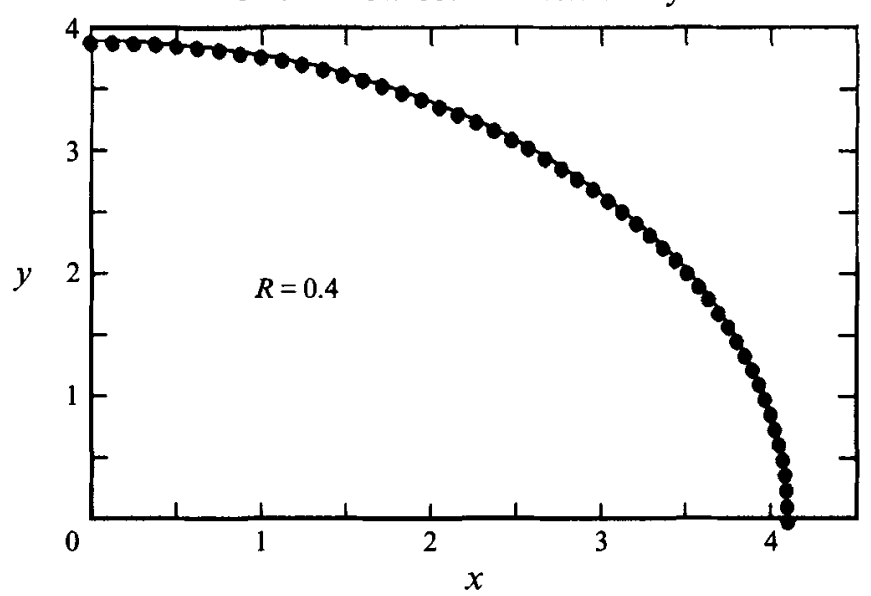

FIGURE 4. Shape of vortex boundary for $M_{\infty}=0, a=0.4$. Solid circles represent

Baker et al. (1976) solution. Solid line is a plot of equations (4.3) and (4.4).

$$
y=\frac{a^{2}-1}{a}\left[\log \left(a^{2}+1+2 a \cos \theta\right)-\log \left(a^{2}+1-2 a \cos \theta\right)\right] .
$$

As shown in figure 4 , Baker et al. parameterize their solutions by the shape ratio $R=P / 2 L$ where $P$ is the perimeter of the vortex and $L$ is the seperation between the vortices. The circulation $\Gamma$ about each vortex is then related to $q_{v}$, the speed of the vortex boundary, by

$$
\Gamma=P q_{v}
$$

Furthermore, at large distances, the array looks like a vortex sheet of strength $2 q_{\infty}$, where

$$
q_{\infty}=\frac{1}{2} \Gamma / L .
$$

Hence, conservation of circulation then requires that $a=R=q_{\infty} / q_{v}$, the location of the singularity in the hodograph plane. Note that the shape ratio is restricted to be in the range $0<R<1$. The limit $R=0$ corresponds to an array of point vortices or a single vortex in unbounded fluid. The opposite limit $R=1$ corresponds to a vortex sheet in which each vortex is pulled out longitudinally and squeezed sideways to lie along a length $L$ of the $x$-axis. The significance of this solution for our problem is that we have obtained the zeroth-order solution of a perturbation solution (the Rayleigh-Jansen expansion) with $M_{\infty}^{2}$ as the small parameter. An examination of the terms of the operator $\mathscr{L}(\psi)$ indicates that compressibility is introduced as regular corrections to the incompressible flow, indicating the possible existence of a regular perturbation expansion in powers of $M_{\infty}$. Below we construct several terms of this solution.

\section{Perturbation solution}

We construct an approximate series solution to the problem depicted in figure 3 valid when $M_{\infty} \ll 1$. The problem is formulated as

$$
q^{2}\left(1-\beta(\gamma-1) q^{2}\right) \psi_{q q}+q\left(1-\beta(\gamma-3) q^{2}\right) \psi_{q}+\left(1-\beta(\gamma+1) q^{2}\right) \psi_{\theta \theta}=0,
$$




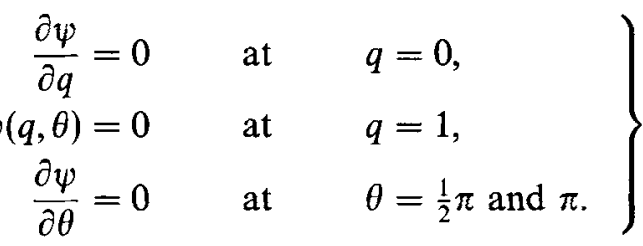

The solution we seek has the Rayleigh-Jansen form,

$$
\psi(q, \theta)=\psi_{o}+M_{\infty}^{2} \psi_{1}+O\left(M_{\infty}^{4}\right)
$$

As noted above, at zeroth order we have the incompressible solution which is given by equation (4.2). The second-order problem is given by

$$
q^{2} \psi_{1_{q q}}+q \psi_{1_{q}}+\psi_{1_{\theta \theta}}=\frac{q^{2}}{a^{2}}\left(\psi_{o_{\theta \theta}}-q \psi_{o_{q}}\right)
$$

with the same boundary conditions as mentioned earlier for equation (5.1). Note that this system has many singular homogenous solutions. According to Prandtl-Glauert theory, we must ensure that the incompressible solution is the dominant singularity as the point at infinity is approached. The only regular homogeneous solution to this problem is the trivial solution $\psi(q, \theta)=0$. Hence, finding a particular solution will then lead to a unique solution to this order. By inspection, a particular solution is given by

$$
\psi_{1_{p}}=\frac{q^{3}}{4} \frac{\partial \psi_{o}}{\partial q}
$$

but closer inspection of the above result shows that this is more singular than the incompressible solution. Hence, we find a complementary function to add to this particular solution such that the final result is regular at the point at infinity and that all boundary conditions are satisfied. This leads to a solution to $O\left(M_{\infty}^{4}\right)$ :

$$
\psi(q, \theta)=\psi_{o}+M_{\infty}^{2} \psi_{1}
$$

where $\psi_{o}$ is given by equation (4.2) and the compressibility correction is given by

$$
\begin{aligned}
\psi_{1}=-q^{2} \frac{\left(a^{4}-1\right)\left(1-q^{2} / a^{2}\right)}{\left(q^{2}+a^{2}+2 a q \cos \theta\right)\left(q^{2}+a^{2}-2 a q \cos \theta\right)} f_{1}(q, \theta) \\
-2 q^{2}\left(1-a^{2}\right) f_{2}(q, \theta)+\left[\frac{1-a^{2}}{a^{2}}\right]
\end{aligned}
$$

where

and

$$
f_{1}(q, \theta)=\frac{a^{4} q^{2}-2 a^{2} q^{4} \cos \theta^{2}+q^{4} a^{2}+a^{2}-2 a^{2} \cos \theta^{2}+q^{2}}{\left(q^{2} a^{2}+1+2 a q \cos \theta\right)\left(q^{2} a^{2}+1-2 a q \cos \theta\right)}
$$

$$
f_{2}(q, \theta)=\frac{q^{2} a^{2}+1-2 \cos \theta^{2}}{\left(q^{2} a^{2}+1+2 a q \cos \theta\right)\left(q^{2} a^{2}+1-2 a q \cos \theta\right)} .
$$

It is essential that the solution above maintains the integrity of our original mapping. We check this by verifying that an integration of the distance traversed around a closed loop in the hodograph plane yields zero. Equivalently, referring to figure 2, we can show that the horizontal distance traversed from $S$ to $B, x_{B S}$, is equal to that traversed going from $\infty_{1}$ to $\infty_{2}, x_{\infty}$. Equation (3.1) gives

$$
\mathrm{d} x=\left[\cos \theta \frac{\partial}{\partial q}\left(\frac{1}{\rho q}\right) \psi_{\theta}-\frac{\sin \theta}{\rho q} \psi_{q}\right] \mathrm{d} q+\left[\frac{\cos \theta}{\rho} \psi_{q}-\frac{\sin \theta}{\rho q} \psi_{\theta}\right] \mathrm{d} \theta
$$


for measuring horizontal distances in the hodograph plane. Hence, the distance from the centre of the vortex to the stagnation point, $x_{B S}$, is given by

$$
x_{B S}=\int_{\pi / 2}^{\pi}\left(\frac{\cos \theta}{\rho} \psi_{q}-\frac{\sin \theta}{\rho q} \psi_{\theta}\right)_{q=1} \mathrm{~d} \theta+\int_{0}^{1}\left(-\frac{\sin \theta}{\rho q} \psi_{q}\right)_{\theta=\pi / 2} \mathrm{~d} q .
$$

This distance must be equal to the length of the streamline at infinity which is given by

$$
x_{\infty}=\lim _{\hat{s} \rightarrow 0} \int_{0}^{-\pi} \mathrm{d} x(\hat{s}, \hat{\delta}) \mathrm{d} \hat{\delta},
$$

where $\hat{s}$ and $\hat{\delta}$ are local polar coordinates centred at the singularity in the hodograph plane. Expanding the above integrals in the Mach number, we find that our perturbation solution, equation (5.5), satisfies the closure condition to $O\left(M_{\infty}^{4}\right)$ automatically. With this solution valid for small $M_{\infty}$, we proceed to discuss the solution at larger values of $M_{\infty}$ which is obtained numerically.

\section{Numerical method}

Since the nature of the singularity is known from our earlier analysis, we opted to solve the following modified but equivalent problem:

$$
\left.\begin{array}{rl}
\psi & =\psi_{s}+\psi_{r}, \\
\mathscr{L}\left(\psi_{r}\right) & =-\mathscr{L}\left(\psi_{s}\right),
\end{array}\right\}
$$

where

$$
\psi_{s}=\log \left[(q-a)^{2}+a^{2} \frac{(\theta-\pi)^{2}}{1-M_{\infty}^{2}}\right]-\frac{a^{2}(\theta-\pi)^{2}}{\left(1-M_{\infty}^{2}\right)(q-a)^{2}+\left(\pi^{2} a^{2} / 4\right)} .
$$

This particular singular form of $\psi_{s}$ is chosen for two reasons. The main advantage is that a local asymptotic analysis near the point at infinity shows that all other higher-order terms vanish in the limit as $(q, \theta) \rightarrow(a, \pi)$. In addition, the top and bottom boundary conditions remain unchanged from those for $\psi$. The remaining boundary conditions for the modified problem are

$$
\left.\begin{array}{rl}
\frac{\partial \psi_{r}}{\partial q} & =-\frac{\partial \psi_{s}}{\partial q}, \quad q=0, \\
\psi_{r}(q, \theta) & =-\psi_{s}(q, \theta), \quad q=1, \\
\frac{\partial \psi_{r}}{\partial \theta} & =0, \quad \theta=\frac{\pi}{2}, \quad \pi .
\end{array}\right\}
$$

The modified problem described above was solved numerically using second-order central differences on a fixed grid

$$
\left.\begin{array}{rl}
q_{i}=(i-1) \Delta q, & i=1, \ldots, N, \\
\theta_{i}=\pi / 2+(j-1) \Delta \theta, & j=1, \ldots, M+1,
\end{array}\right\}
$$

where $\Delta q=1 /(N-1)$ and $\Delta \theta=\pi /(2 M)$, and we put $\psi_{i, j}=\psi_{r}\left(q_{i}, \theta_{j}\right)$. The finite difference form of equation (6.1) is given by

$$
\begin{aligned}
\left(\frac{A_{i, j}}{\delta q^{2}}+\frac{B_{i, j}}{2 \delta q}\right) \psi_{i+1, j}-2\left(\frac{A_{i, j}}{\delta q^{2}}+\frac{C_{i, j}}{\delta \theta^{2}}\right) \psi_{i, j} & +\left(\frac{A_{i, j}}{\delta q^{2}}-\frac{B_{i, j}}{2 \delta q}\right) \psi_{i-1, j} \\
& +\frac{C_{i, j}}{\delta \theta^{2}}\left(\psi_{i, j+1}+\psi_{i, j-1}\right)=f_{i, j}
\end{aligned}
$$




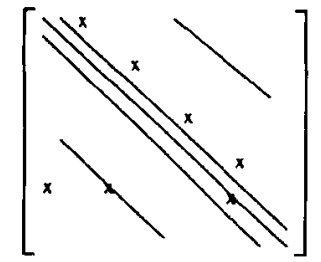

A

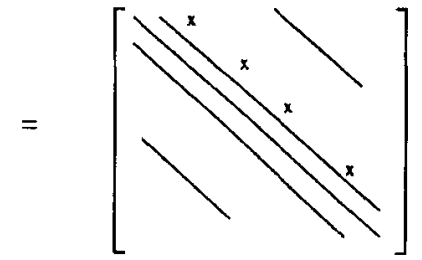

$\hat{\boldsymbol{A}}$

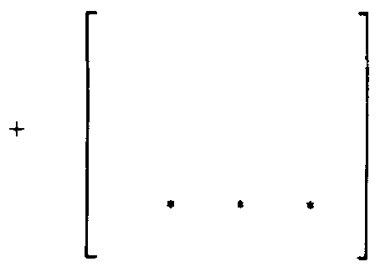

$U \cdot V^{T}$

FigURE 5. Decompostion of finite difference coefficient matrix. The cross and asterisks symbols emphasize the location of the corrected rows. Matrix size is $(N \cdot(M+1) \times N \cdot(M+1))$.

where $f_{i, j}=-\mathscr{L}\left(\psi_{s}\left(q_{i}, \theta_{j}\right)\right)$ and the coefficients are defined as follows:

$$
A_{i, j}=q_{i}^{2}\left(1-\beta(\gamma-1) q_{i}^{2}\right), \quad B_{i, j}=q\left(1-\beta(\gamma-3) q_{i}^{2}\right), \quad C_{i, j}=\left(1-\beta(\gamma+1) q_{i}^{2}\right) .
$$

At the top and bottom boundaries, where $\theta=\pi$ and $\theta=\pi / 2$, the derivative conditions are satisfied by adding fictitious points parallel to these boundaries and applying centred differences except for the point at $\left(q_{i}, \theta_{j}\right)=(a, \pi)$. We cannot use ghost points there because the forcing term in equation (6.1) is not defined. Hence, since we are solving for $\psi_{r}$, which is the regular part of our solution, we found it sufficient to impose the derivative condition using forward differencing:

$$
\hat{i}=\frac{a}{\Delta q}+1, \quad-3 \psi_{\hat{i}, M+1}+4 \psi_{i, M}-\psi_{i, M-1}=0,
$$

where $\hat{i}$ is the location of the singularity on the grid.

The use of forward differencing at $q=0$ also proved to be the most effective means for avoiding unphysical $\theta$-dependence of the stagnation value. Implementation of the right-hand-side Dirichlet condition $\left(\psi_{r}(1, \theta)=-\psi_{s}(1, \theta)\right)$ is straightforward. Finally, the problem is reduced to one of linear algebra and solving a system $\boldsymbol{A} \cdot \boldsymbol{x}=\boldsymbol{B}$. At this stage, the main difficulty is resolution. Figure 5 shows a schematic of the banded coefficient matrix $\boldsymbol{A}$ and its decomposition. If not for the special treatment of the singular point, $A$ would have been amenable to transform methods, hence, we chose to use the capacitance matrix approach to change $A$ appropriately. The decomposition of matrix $\boldsymbol{A}$ can be written as

$$
\boldsymbol{A}=\hat{\boldsymbol{A}}+\boldsymbol{U} \cdot \boldsymbol{V}^{T} \text {. }
$$

The Woodbury formula, which is the block-matrix version of the Sherman-Morrison formula (Golub \& Van Loan 1989), relates $\mathbf{A}^{-1}$ to its decompostion as

$$
\left(\hat{\boldsymbol{A}}+\boldsymbol{U} \cdot \boldsymbol{V}^{T}\right)^{-1}=\hat{\boldsymbol{A}}^{-1}-\left[\hat{\boldsymbol{A}}^{-1} \cdot \boldsymbol{U} \cdot\left(1+\boldsymbol{V}^{T} \cdot \hat{\boldsymbol{A}}^{-1} \cdot \boldsymbol{U}\right)^{-1} \cdot \boldsymbol{V}^{T} \cdot \hat{\boldsymbol{A}}^{-1}\right],
$$

where the term $\left(1+\boldsymbol{V}^{T} \cdot \hat{\boldsymbol{A}}^{-1} \boldsymbol{A} \cdot \boldsymbol{U}\right)^{-1}$ is known as the capacitance matrix and it has dimensions $(p \times p)$ where $p$ is the number of corrected rows. Since we have Neumann boundary conditions in the $\theta$-direction, we apply a discrete cosine transform on $\hat{\boldsymbol{A}}$, reducing our problem to a set of $k$ differential equations in $q$ where $k$ is the Fourier mode. At this stage, we choose to apply the Woodbury formula one more time so that at each mode we have a tridiagonal matrix. Note that the extra entry in the first row of each of the $k$-equations comes from our choice of forward differencing at $q=0$. Making this extra effort increases our resolution compared to inverting the original banded matrix $\boldsymbol{A}$.

Note that in obtaining $\psi_{r}$, we solve a linear system. This is not obvious at the outset. Indeed Moore \& Pullin (1987) find residual nonlinearity in their formulation 


\begin{tabular}{ccc}
$q$ & \multicolumn{2}{c}{$\psi(q, \theta=\pi / 2)$} \\
\cline { 2 - 3 } 0.00 & Numerical & Perturbation \\
0.20 & -3.6129 & -3.6127 \\
0.40 & -2.2796 & -3.1784 \\
0.80 & -0.6199 & -2.2796 \\
1.00 & 0.0000 & -0.6197 \\
& & 0.0000
\end{tabular}

TABLE 1. A comparison of the numerical and perturbation results at $M_{\infty}=0.1$ and $a=0.4$

of the compressible vortex pair problem in the form of a forced closure of the physical plane when reconstructed from the solution of the hodograph problem. This led us to expect an iterative solution procedure resulting from the non-linear nature of compressible flow problems, yet the transformation to the hodograph plane in this specific example has lead to an entirely linear problem. Our final solution is written as

$$
\psi(q, \theta)=\psi_{s}(q, \theta)+\psi_{r}(q, \theta) .
$$

For $M_{\infty} \ll<1$, we compare our numerical solution with our perturbation solution for various values of $q$ and $\theta$ and successfully verify that the error is $O\left(M_{\infty}\right)^{4}$. Table 1 shows the results from one such test.

\section{Results}

We perform a parameter search to determine those solutions that are physically relevant. It is well known (Landau \& Lifschitz 1959) that if the Jacobian of the hodograph transformation vanishes at any point, the solution will exhibit cuspidal behaviour corresponding to the onset of limit lines (Kuo \& Sears 1954). Hence in our search of the parameter space $\left(a, M_{\infty}\right)$, we monitored the Jacobian for changes of sign. The search was done at a fixed value of $a$, stepping in the Mach number in increments of 0.001 , on a $(N, M+1)=(500 \times 128)$ grid, until a limit line was approached. Note that the computation was stopped at the first sight of a limit line and then the resolution was increased to $(N, M+1)=(1000 \times 256)$ and $(2000 \times 256)$ to ensure that the results are grid independent. The level of accuracy of these computations was up to six digits. The outcome of this investigation is depicted in figure 6 which summarizes the range of solutions found here. It is evident from the figure that transonic shock-free flows continuous in the $\left(a, M_{\infty}\right)$ parameter space can and do exist for our problem.

\subsection{Evacuated vortex core}

The dotted line in figure 6 is an upper boundary on the supersonic flow region. It actually corresponds to the limit when the pressure in the core of the vortex is reduced to zero. This evacuated core limit is a special case since our problem then reduces to a single-parameter family. Recalling the energy equation (2.2), we note the following:

$$
\frac{c_{v}^{2}}{\gamma-1}+\frac{1}{2} q_{v}^{2}=\frac{c_{\infty}^{2}}{\gamma-1}+\frac{1}{2} q_{\infty}^{2}
$$




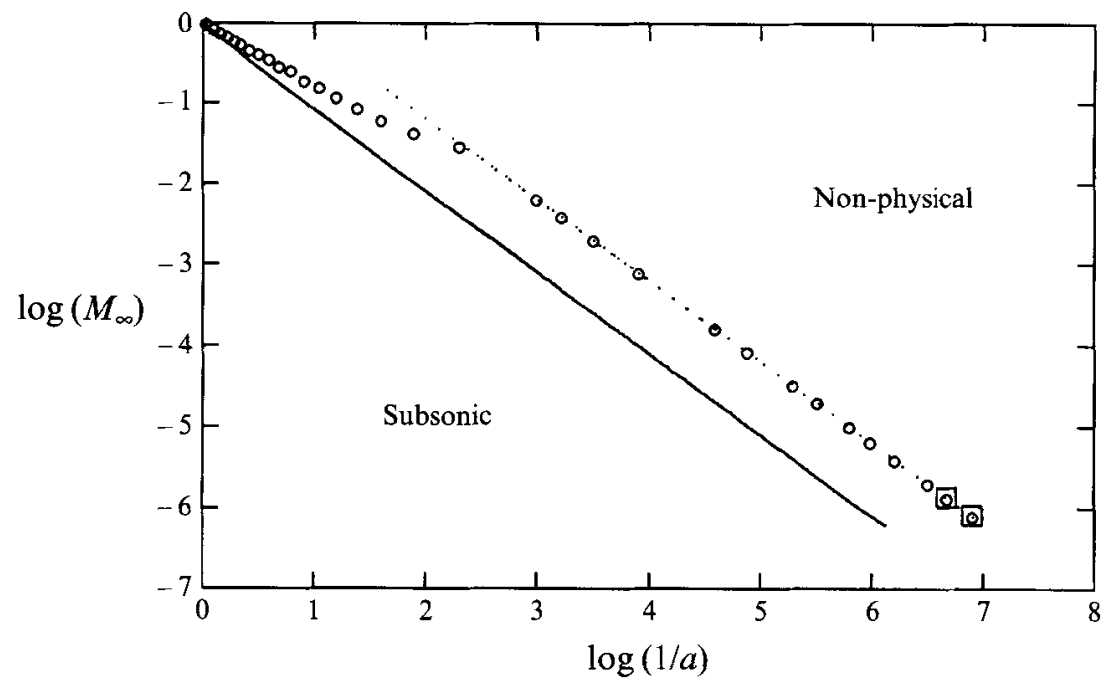

FIGURE 6. The solid line represents the loci of sonic vortices, $M_{v}=1.0$. The dotted line indicates the evacuated vortex core limit, $p_{v}=0$, o represents a change of sign of the Jacobian and $\square$ indicates no change of sign.

Since the core is at vacuum, $c_{v}=0$, manipulating the above equation gives a relationship for $M_{\infty}$ in terms of $a$,

$$
M_{\infty}=\left(\frac{2}{\gamma-1} \frac{a^{2}}{1-a^{2}}\right)^{1 / 2} .
$$

In relation to figure 6 , this corresponds to the dotted line. We chose $a$ as the parameter for this search and found that limit lines appeared quite early. Actually, the largest value attained before the Jacobian of the transformation changed sign was $a=0.0015$ corresponding to $M_{\infty}=0.00335$. Note that in order to achieve good resolution of the solution near $q=0$, where we have a Neumann condition, we had to use fine grids. The evacuated vortex results were computed on $(N, M+1)=(2000 \times 128),(4000 \times 128)$ and $(4000 \times 256)$ grids. At this point it was necessary to determine the validity of the two solutions for which there was no change of sign of the Jacobian. Hence, we searched for the minimum of the Jacobian and monitored its value as we increased the resolution. Figure 7 clearly shows that as the resolution is increased, the Jacobian tends to zero. This leads us to conclude that there exists no evacuated vortex solution for this problem free of limit lines.

Following Moore \& Pullin 1987, we find a leading-order approximation to the value of the stream function at the boundary of the evacuated vortex by the method of matched asymptotic expansions. The incompressible solution, Lamb (1932), is an outer solution which is matched to the solution for an isolated hollow vortex, Thompson (1972), giving

$$
\left.\begin{array}{rl}
\psi_{v} & \left.=\frac{\Gamma}{4 \pi}\left[F(1 ; \gamma)-\log a^{2}\right)\right], \\
F(s ; \gamma) & =\frac{1}{\gamma-1} \int_{0}^{s} \log \xi(1-\xi)^{\frac{2-\gamma}{\gamma-1}} \mathrm{~d} \xi .
\end{array}\right\}
$$

In table 2 we compare the asymptotic result for the vortex boundary with that from our numerical solution. 


\begin{tabular}{ccc}
$M_{\infty}$ & \multicolumn{2}{c}{$\psi_{v}$} \\
\cline { 2 - 3 } & Numerical & Asymptotic \\
0.225 & -5.8946 & -5.84959 \\
0.112 & -8.6342 & -8.62218 \\
0.045 & -12.2892 & -12.28734 \\
0.022 & -15.0600 & -15.0599 \\
0.00335 & -22.6540 & -22.6484
\end{tabular}

TABLE 2. A comparison of the numerical and asymptotic results for the evacuated vortex

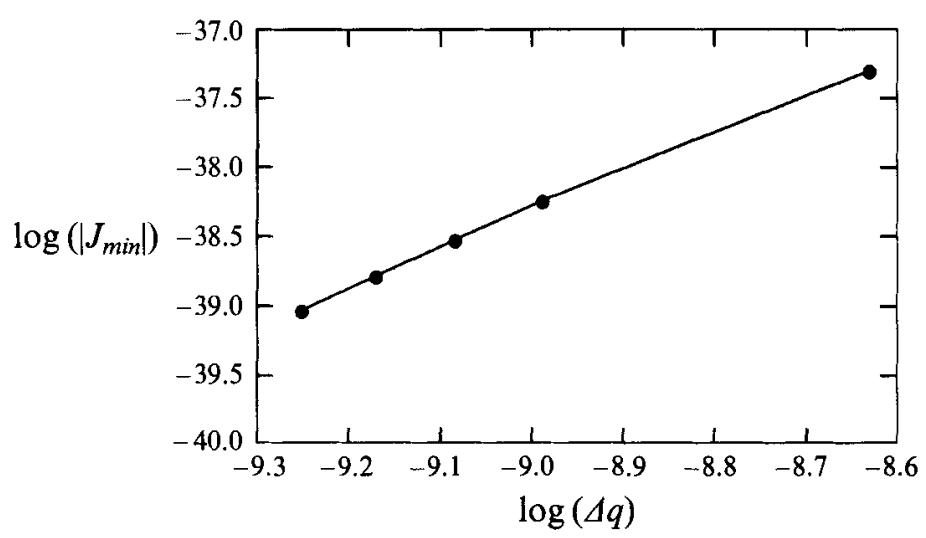

FIGURE 7. The absolute value of the minimum of the Jacobian is plotted versus different mesh sizes. $M=256$ is kept constant as $\Delta q$ is decreased.

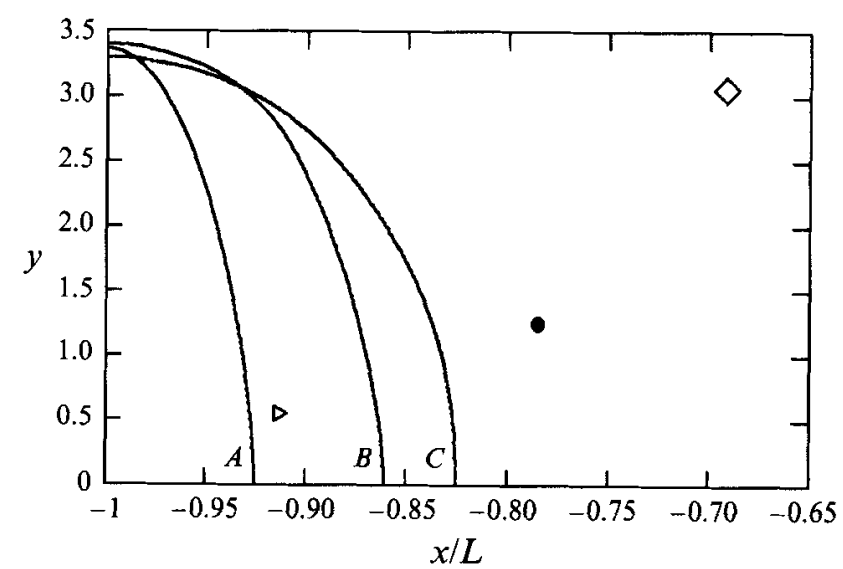

FIGURE 8. Vortex boundaries and the position of the corresponding limit lines. Boundary $\mathrm{A}$ and $\triangleright$ correspond to $\left(a, M_{\infty}\right)=(0.1,0.215)$. Boundary $\mathrm{B}$ and $\bullet$ correspond to $\left(a, M_{\infty}\right)=(0.2,0.296)$. Boundary $\mathrm{C}$ and $\diamond$ correspond to $\left(a, M_{\infty}\right)=(0.25,0.343)$.

\subsection{Streamlines and limit lines}

While monitoring the Jacobian for a change of sign, we also noted the exact location on the grid at which the limit lines first appear. Figure 8 displays a few examples. We have plotted the boundary of the vortex and the position of the first occurrence of a limit line for a given $\left(a, M_{\infty}\right)$.

In figure 9, we display the free boundaries for a progression of vortices ranging 


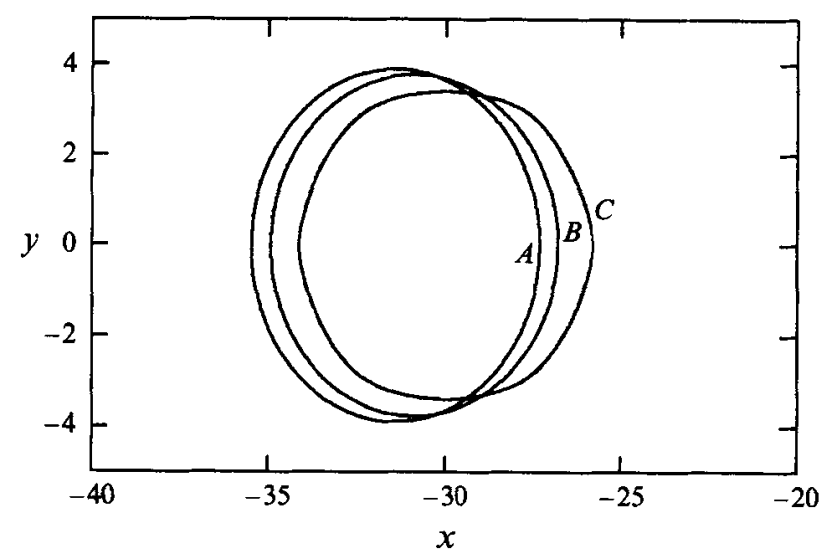

FIGURE 9. Incompressible, sonic and supersonic vortices. $a=0.2$ fixed and $M_{\infty}$ is increased. The Mach number on the vortex boundaries is $M_{v_{A}}=0, M_{v_{B}}=1.00$ and $M_{v_{C}}=1.933$. The distance $x$ is measured from the stagnation point.

from incompressible to subsonic to supersonic flow in the range of the $\left(M_{\infty}, a\right)$ space where no limit lines were detected. Note that for the free boundaries shown, the shape ratio was held fixed, $a=0.2$. We see that as $M_{\infty}$ is increased, the vortices shrink in size and get closer together. It is intuitive to reason that the flow is being compressed but, actually, there exists some experimental evidence for such occurrences. M. G. Mungal, J. C. Hermanson \& P. E. Dimotakis have produced some unpublished schlieren data which shows that the large-scale structures in a shear layer shrink in size as the flow velocity is increased. For photos and discussion refer to Dimotakis (1991). Note that the present analogy is only qualitative.

In figure 10, we have plotted the streamlines of a transonic flow in the physical plane. The dotted line on the figure denotes the sonic line. Note that the sonic line is closer to the vortex boundary in the $x$-direction than in the $y$-direction. This phenomenon is explained by the fact that the flow in the $x$-direction is required to reach a stagnation point.

\subsection{Vortex geometry}

At this point, we return to figure 7 to identify the range of $\left(a, M_{\infty}\right)$ for which solutions exist and survey this parameter space for detailed knowledge of the possible vortex boundary geometries. Figure 11 shows the relevant length scales in the physical plane.

Note that $b_{1}$ and $b_{2}$ are measured from the centre of the vortex. $\lambda(=L / 2)$ measures the distance from the centre of the vortex to the stagnation point. We found that monitoring the aspect ratio of the vortex boundary, $b_{1} / b_{2}$, and the parameter $b_{1} / \lambda$ was sufficient to give a complete overall view of all possible geometrical configurations that the vortices attain at different values of $\left(a, M_{\infty}\right)$.

Figure 12 shows that for a fixed value of $a$ the vortices shrink and get closer together as the Mach number is increased. Note that $b_{1} / b_{2}=1$ is the limit of circular vortices. Figure 13 shows the extent of the vortex boundary stretched in the horizontal direction. It is evident that for a fixed $a$, as the Mach number is increased, the vortex boundary stretches out in the horizontal direction.

When $b_{1} / \lambda=0$, we are at the point vortex solution whereas $b_{1} / \lambda=1$ is the limit of the shear layer solution. Note that in both figure 12 and figure 13 the solutions were terminated at the first occurrence of a limit line. 


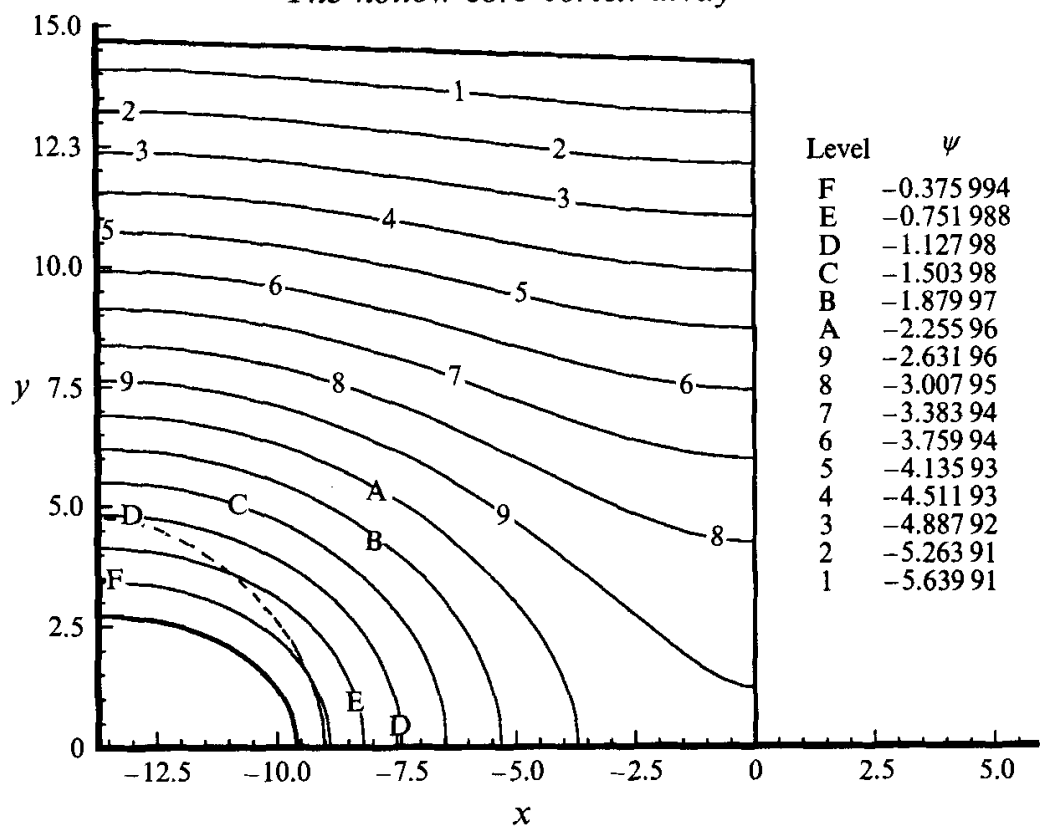

FIGURE 10. Streamlines for a transonic shock-free flow. $a=0.4$ and $M_{\infty}=0.48$. Here, $M_{v}=1.4$, where $M_{v}$ is the Mach number on the vortex boundary indicating supersonic flow. The dotted line indicates the sonic line.

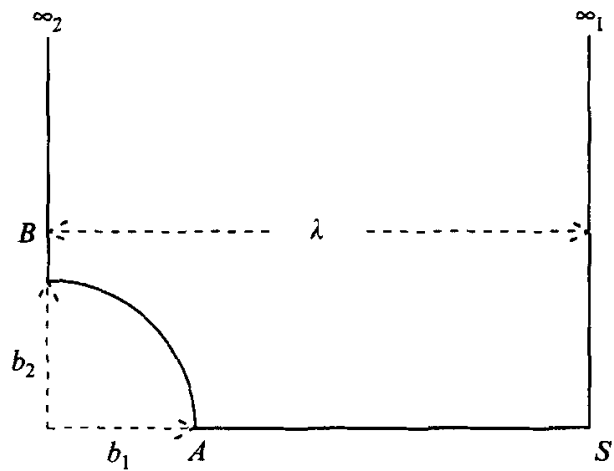

FIGURE 11. A description of the relevant length scales of the problem.

\section{Conclusion}

We have shown that it is possible to have steady flow of an infinite, periodic array of vortices in a compressible flow, and furthermore, that this array can exhibit transonic shock-free flow. It has also been illustrated that by taking special care in treating the singularity of the transformation at infinity, it is possible to reduce a compressible transonic flow to a strictly linear problem in the hodograph plane. The evacuated vortex core results indicate that such solutions can be constructed in the hodograph plane but that they all exhibit cuspidal behaviour which indicates the onset of limit lines.

Further extensions of this work include investigation of the stability of this vortex array. We have shown that steady solutions exist and now we must determine whether these solutions are stable. Note that the vorticity in this problem was concentrated into vortex sheets on the surfaces of the vortices. It would be interesting to investigate the case where the vorticity is continously distributed throughout the whole flow. Also 


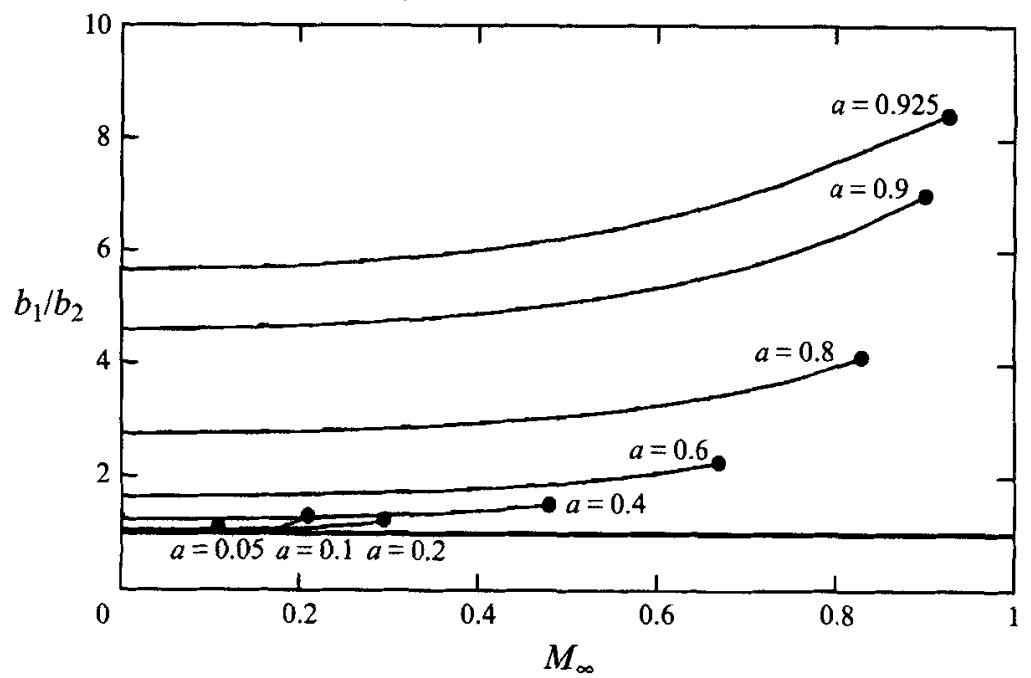

FIGURE 12. Aspect ratio of the vortex boundary for all possible solutions. Heavy straight line represents the limit of circular vortices, $\bullet$ indicates the occurrence of a limit line.

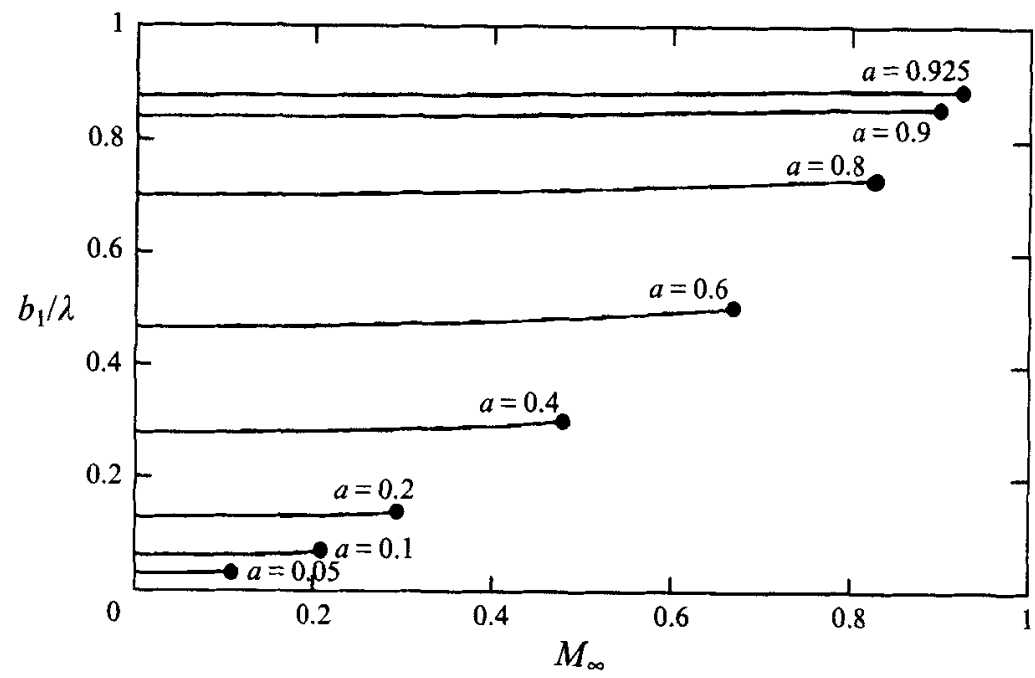

FIGURE 13. Stretching of the vortex boundary in the $x$-direction, - indicates the occurrence of a limit line.

of interest is to determine whether there exists a class of compressible free boundary problems that lend themselves to a linear system of equations in the hodograph plane.

\section{REFERENCES}

BAKER, G. R., SAFFMAN, P. G. \& SHEFFIELD, J. S. 1976 Structure of a linear array of hollow vortices of finite cross section. J. Fluid Mech. 74, 469-476.

Brown, S. N. 1965 The compressible leading-edge vortex. J. Fluid Mech. 22, 17-32.

Dimotakis, P. E. 1991 Turbulent free shear layer mixing and combustion. Prog. Astro. Aero. 137, 265-340.

Golub, G. H. \& Van LoAn, C. F. 1989 Matrix Computations. The Johns Hopkins University Press. HuANG, M. K. \& CHOw, C. Y. 1982 Trapping of a free-vortex by Joukowski airfoils. AIAA J. 20, 292-298. 
Kuo, Y. H. \& SeARS, W. R. 1954 Plane subsonic and transonic potential flows. In General Theory of High-Speed Aerodynamics: Vol. VI, High Speed Aerodynamics and Jet propulsion (ed. W. R. Sears), pp. 490-577. Princeton University Press.

Lamb H. 1932 Hydrodynamics. Cambridge University Press.

Landau, L. D. \& Lifshitz, E. M. 1959 Fluid Mechanics. Pergamon Press.

MaCK, L. M. 1960 The compressible viscous heat-conducting vortex. J. Fluid Mech. 8, 284-292.

Milne-Thomson, L. 1966 Theoretical Aerodynamics. Macmillan.

MoORE, D. W. 1985 The effect of compressibility on the speed of a vortex ring. Proc. R. Soc. Lond. A 397, 87-97.

MoORE, D. W. \& Pullin, D. I. 1987 The compressible vortex pair. J. Fluid Mech. 185, 171-204.

RINGLEB, F. 1940 Exakte Losungen der Differentialgleichungen einer adiabatischen Gasstromung Z. Angew. Math. Mech. 20(4), 185-198. (Available as R.T.P. Translation No. 1609, British Ministry of Aircraft Production.)

Roshкo, A. 1976 Structure of turbulent shear flows: a new look. AIAA J. 14, 1349-1357.

Saffman, P. G. \& Sheffield, J. S. 1977 Flow over a wing with an attached free vortex. Stud. Appl. Maths 57, 107-117.

Shapiro, A. H. 1953 The Dynamics and Thermodynamics of Compressible Flow, Vol I. John Wiley and Sons.

Thompson, P. A. 1972 Compressible Fluid Dynamics. McGraw-Hill.

Von Mises, R. 1958 Mathematical Theory of Compressible Fluid Flow. Academic Press. 